\title{
Preparing Irradiated and Marked Male Aedes aegypti Mosquitoes for Release in an Operational Sterile Insect Technique Program
}

\author{
Bianca J. Moreno ${ }^{1}$, Robert L. Aldridge ${ }^{1}$, Seth C. Britch ${ }^{1}$, Barbara E. Bayer ${ }^{1}$, Jedidiah Kline ${ }^{1}$, Daniel A. Hahn ${ }^{2}$, Chao \\ Chen $^{2}$, Kenneth J. Linthicum ${ }^{1}$ \\ ${ }^{1}$ US Department of Agriculture, Agricultural Research Service Center for Medical, Agricultural, \& Veterinary Entomology ${ }^{2}$ Department of Entomology and \\ Nematology, University of Florida
}

\section{Corresponding Author}

Seth C. Britch

Seth.Britch@usda.gov

\section{Citation}

Moreno, B.J., Aldridge, R.L., Britch, S.C., Bayer, B.E., Kline, J., Hahn, D.A.,

Chen, C., Linthicum, K.J. Preparing Irradiated and Marked Male Aedes aegypti Mosquitoes for Release in an Operational Sterile Insect Technique Program. J. Vis. Exp. (169), e62260, doi:10.3791/62260 (2021).

\section{Date Published}

March 12, 2021

DOI

$10.3791 / 62260$

URL

\section{Abstract}

The control of such human diseases as dengue, Zika, and chikungunya relies on the control of their vector, the Aedes aegypti mosquito, because there is no prevention. Control of mosquito vectors can rely on chemicals applied to the immature and adult stages, which can contribute to the mortality of non-targets and more importantly, lead to insecticide resistance in the vector. The sterile insect technique (SIT) is a method of controlling populations of pests through the release of sterilized adult males that mate with wild females to produce non-viable offspring. This paper describes the process of producing sterile males for use in an operational SIT program for the control of Aedes aegypti mosquitoes. Outlined here are the steps used in the program including rearing and maintaining a colony, separating male and female pupae, irradiating and marking adult males, and shipping Aedes aegypti males to the release site. Also discussed are procedural caveats, program limitations, and future objectives.

\footnotetext{
jove.com/video/62260
}

\section{Introduction}

Transmission of mosquito-borne pathogens to humans causes millions of cases of disease and deaths each year worldwide. In the absence of effective, approved vaccines for mosquito-borne diseases, such as Zika or dengue fever, one of the most effective ways to reduce transmission is to reduce disease-vector mosquito populations. Vexingly, an increasing number of mosquito species, traditionally targeted by pesticides, are displaying increasing levels of pesticide resistance ${ }^{1}$. Simultaneously, government agencies have aggressively deregistered or banned previously approved pesticides, and few new, effective chemical control measures are being developed ${ }^{2,3}$. This constellation of obstacles to 
mosquito control has motivated the exploration of alternative non-chemical techniques to reduce mosquito populations.

Certain mosquito species present challenges to control issues of resistance and pesticide registration. Aedes aegypti (L.) is a prominent disease-vector mosquito that is extremely difficult to control through traditional integrated vector management due to the cryptic peridomestic habitat exploited by this species for immature development and adult resting ${ }^{4,5}$. Challenges related to the exploitation of the cryptic habitat around residences include the difficulty of reaching these locations with pesticide spray techniques as well as the potential lack of acceptance by the public for repeated access to private property for public health vector control agencies to conduct the intensive surveillance and control activities crucial to effective integrated vector management (IVM) for this species.

Fortunately, SIT, an approach proven successful for enduring control of other highly challenging insect species ${ }^{6}$, is being applied to the Aedes aegypti problem in a groundbreaking series of experiments and operational trials based in St. Augustine, Florida (KJL, RLA, SCB unpublished data). SIT has been applied to a range of insect species, including mosquitoes, and has been reviewed in depth ${ }^{7,8}$. SIT leverages the mass release of colony-reared males sterilized, for example, by exposure to ionizing radiation or chemicals to overwhelm the mate choice of natural populations of females. Sterilized males that mate with wild females render the eggs infertile due to damage suffered by male gametes, and if present in sufficient numbers, can theoretically crash the natural Aedes aegypti population.

An SIT program was initiated to attempt to reduce populations of Aedes aegypti in an urban area in Atlantic coastal Florida where this species recently re-colonized and is expanding and presenting a public health risk for transmission of viruses such as Zika, dengue, or chikungunya. To maximize the potential for compatibility with wild females, a new colony was established using wild-caught Aedes aegypti from the target population to produce males for the program ${ }^{9}$. This was based on the hypothesis that locally derived, colony-reared males would be more likely to be competitive with local wild males for mating with local wild females. For the SIT to be effective, not only do overwhelming numbers of sterile males need to be present in the target area, but they also have to be capable of effectively courting and mating with local wild female mosquitoes.

A series of experiments was conducted to determine the optimal number of sterile males to release (KJL, RLA, SCB unpublished data) as well as optimal doses of radiation that would render the males infertile without interfering with survival, behavior, or acceptance by wild females (KJL, RLA, SCB unpublished data). These data are forthcoming in allied publications from this group, but some of these findings are captured in this protocol as well and could be used as a starting point for new SIT Aedes aegypti control programs elsewhere. This species is constantly expanding its range, and SIT programs show great promise to be cost-effective, long-term solutions to control this population. The objective of this protocol is to produce sterilized, male, colony-reared Aedes aegypti mosquitoes for systematic release into outdoor areas to disrupt the natural reproductive cycles of local Aedes aegypti populations in an operational public health vector control program.

While similar protocols and workflows have been published for the production of transgenic Aedes aegypti males and production workflows for Aedes SIT, or Wolbachiabased incompatibility programs have been published 
elsewhere, this protocol illustrates how existing protocols have been adapted for Aedes aegypti production, separation and irradiation of male pupae, marking and packaging adult males, and shipment to the release site for this program $9,10,11,12,13,14,15,16,17,18$. The marking component of this protocol may not be required in a mature operational SIT program; however, it has been included here because it is one way to monitor the efficacy and control the quality of the entire process in the early years of establishing the SIT program. Mosquito control programs are typically run by local authorities, so they can vary widely in many aspects of their organization from size and funding base to tuning control tactics to maximize local success. Thus, the protocol described herein should be evaluated for compatibility with available resources.

\section{Protocol}

NOTE: This protocol is specific to the handling of Aedes aegypti but may be modified to be effective for other mosquito species.

\section{Production and maintenance of an Aedes aegypticolony}

1. Rear adult Aedes aegypti and produce eggs.

1. Prepare a $0.6 \mathrm{~m} \times 0.6 \mathrm{~m} \times 0.6 \mathrm{~m}$ collapsible, aluminum frame, large rearing cage with $20 x$ 20 fiberglass mesh screening and a reach-in stockinette sleeve on one vertical wall.

2. Place a $1900 \mathrm{~mL}$ plastic tub with Aedes aegypti pupae (1:1 sex ratio) in each rearing cage, tie the sleeve closed, and leave cups in place for eclosion until no more adults emerge (i.e., approximately 4 days). At this time, remove the cups, and maintain the adult rearing cages at $28-30{ }^{\circ} \mathrm{C},>50 \%$ relative humidity (Rh), and a 12:12 or 14:10 light:dark (L:D) cycle.

NOTE: Production of Aedes aegypti pupae is described in section 1.2. The density of pupae in the $1900 \mathrm{~mL}$ tub should be such that there is enough room for all pupae to come up for air simultaneously.

3. Twenty-four hours after the pupae are placed in the rearing cages, place a container of $10 \%$ sucrose solution with a sponge wick, and suspend a 10 $\mathrm{cm} \times 2 \mathrm{~cm}$ sponge soaked in honey from a wire hook in each cage to provide separate sources of hydration and nutrition to adult mosquitoes. Monitor the sponges and sucrose container for dryness or mold growth, and replenish or change as needed.

NOTE: Use a $120 \mathrm{~mL}$ plastic cup with a $10 \mathrm{~cm} \times 2$ $\mathrm{cm}$ sponge wick fitted through a cutout in the lid in small rearing cages and a $460 \mathrm{~mL}$ plastic cup with a $12 \mathrm{~cm} \times 8 \mathrm{~cm}$ sponge wick in large cages.

4. Provide a blood meal to each rearing cage $48-72$ $\mathrm{h}$ after the majority of adults have emerged and every 2-3 days thereafter to maintain high numbers of bloodfed females to maximize egg yield. Fill a lambskin condom with $50-100 \mathrm{~mL}$ of defibrinated bovine blood, and warm to approximately $37^{\circ} \mathrm{C}$ in a hot water bath. Then, use a cloth or paper towel to pat down and partially dry the condom before placing it on a paper-lined Petri dish inside the cage for 30-60 min.

NOTE: Before use, rinse the inside and outside of each condom with water 2-3x to remove lubricants or other substrates, and check for holes. Condoms can be reused for 3-5 feedings by rinsing out the blood and storing them in a cup of cool water. As some 
colonies may experience ant infestations, condoms may need to be suspended to limit access.

5. Wait 48-72 $\mathrm{h}$ after each bloodfeeding, and then introduce an oviposition cup into each adult rearing cage. Prepare the oviposition cups by adding 200 $\mathrm{mL}$ of filtered pupal water (i.e., the water larvae and pupae were reared in) into a plastic $460 \mathrm{~mL}$ cup furnished with an $8-10 \mathrm{~cm}$ high $\times 30 \mathrm{~cm}$ wide sheet of seed germination paper (oviposition paper) fitted flush along the inner circumference of the cup. Check the oviposition papers daily, replace them every 2-4 days, and carefully store the egg-laden oviposition papers by leaving them to dry for $24-48$ h at $>50 \% \mathrm{Rh}^{19}$.

NOTE: Leave the oviposition cups in the rearing cages for no more than $72 \mathrm{~h}$ to prevent the eggs from hatching.

6. Maintain the adult rearing cages for up to $3-4$ weeks before breaking them down and setting up new adult rearing cages.

1. To break down a rearing cage, remove and clean the oviposition cup, store the eggladen oviposition paper, remove and clean the sucrose solution container and honey sponge, and freeze the cage to kill all mosquitoes.

2. Remove all mosquito bodies, and thoroughly clean the inside and outside of each cage with dilute soap and water using paper towels and scouring pads with sponges. Allow the clean cage to dry for at least $24 \mathrm{~h}$ before using it in the next rearing cycle.

NOTE: Use vacuum equipment fitted with high-efficiency filtration to remove particulates that can lead to allergies. Sucrose solution containers can be cleaned and reused 3-5x.

2. Rearing Aedes aegypti larvae from eggs

1. Prepare a stock of larval nutritional slurry by mixing $80 \mathrm{~g}$ of a 3:2 ratio of bovine liver powder:brewer's yeast in $2200 \mathrm{~mL}$ tap water. Prepare pulverized fish food. Pour fish flakes into a spice grinder and grind until it is a fine powder.

NOTE: This slurry is designated as brown in this laboratory.

2. Using egg-laden (5,000-10,000 eggs) oviposition paper from step 1.1.5, cut a 3-7 $\mathrm{cm}$ portion of the egg paper perpendicular to the oviposition line, and place it in a $460 \mathrm{~mL}$ container half-filled with tap water along with a pinch of pulverized fish food flakes. Cover and agitate vigorously for at least $1 \mathrm{~min}$.

NOTE: Egg paper must be stored for at least 7 days (but no more than 90 days, which can reduce hatching) post-oviposition before initiating the hatching process to permit embryonation. Bacteria and algae present in the fish food rapidly deoxygenate the water, which triggers larval development.

3. Pour the entire contents of the container from step 1.2.2 into a larval rearing pan prepared with $3 L$ of tap water and $50 \mathrm{~mL}$ of brown slurry. Mark the pan with start date, strain information, the feeding schedule per Table 1, and store at $28-30{ }^{\circ} \mathrm{C},>50 \% \mathrm{Rh}$, and a 12:12 or 14:10 L:D cycle.

NOTE: The $3 \mathrm{~L}$ of water to $50 \mathrm{~mL}$ of brown ratio is based on the depth of water in the particular larval pans mentioned in the Table of Materials. Different size pans will support different pupal densities and 
thus require different amounts of water and brown slurry. Larval feeding rates in Table $\mathbf{1}$ are given as a range; selection of the amount used is based on experience and determination of the overall health of the developing larvae using variables such as water turbidity, color, and odor; presence of bacterial film on the water; number or ratio of live and dead larvae; and the motility of larvae. On days 3 to 6 , feed immature mosquitos pulverized fish food according to Table 1. Adding water, reducing food, and setting up 2-3 extra pans than the project requires are ways to manage unhealthy larval pans.

\begin{tabular}{|c|c|c|c|}
\hline Day & $\begin{array}{l}\text { Volume nutritional } \\
\text { slurry added }\end{array}$ & Volume water added & Actions \\
\hline 1 & $50 \mathrm{~mL}$ (slurry) & $3000 \mathrm{~mL}$ & \\
\hline 2 & (no food) & (no water) & \\
\hline 3 & $\begin{array}{c}1 / 4-1 / 2 \text { tsp } \\
\text { (pulverized fish food) }\end{array}$ & $500-1000 \mathrm{~mL}$ & \\
\hline 4 & $\begin{array}{c}1 / 2-3 / 4 \text { tsp } \\
\text { (pulverized fish food) }\end{array}$ & $500-1000 \mathrm{~mL}$ & \\
\hline 5 & $\begin{array}{c}1 / 2-3 / 4 \text { tsp } \\
\text { (pulverized fish food) }\end{array}$ & $500-1000 \mathrm{~mL}$ & \\
\hline 6 & $\begin{array}{c}1 / 4-1 / 2 \text { tsp } \\
\text { (pulverized fish food) }\end{array}$ & $500-1000 \mathrm{~mL}$ & \\
\hline 7 & (no food) & (no water) & strain pupae and larvae \\
\hline
\end{tabular}

Table 1: Feeding schedule for mass rearing of Aedes aegypti larvae.

\section{Separation of male Aedes aegyptipupae}

1. Concentrate pupae from the larval pans. Once the approximate threshold proportion of pupae is reached, pour the contents of each pan through a sieve (size 20-40). Use a squeeze bottle of tap water to wash the pupae and larvae out of the sieve into a $3000 \mathrm{~mL}$ graduated plastic beaker.

NOTE: Only 2-3 larval pans should be transferred to each $3000 \mathrm{~mL}$ beaker to avoid overcrowding so that pupae may reach the surface comfortably. Pupae are expected to develop between $130-140 \mathrm{~h}$ after egg hatch under the temperature and light conditions mentioned in step 1.2.3. Expect noticeable egg hatch on the same day that the eggs are set up. Depending on the environmental conditions, approximately $20-70 \%$ of the larvae will have pupated and be ready to be sieved within 6 days. Partitioning the pupae across multiple $3000 \mathrm{~mL}$ beakers ensures manageable volumes to pour into the separator.

2. Separate male pupae from the larvae and female pupae. 
NOTE: This step can be performed by one operator or two operators.

1. For a single operator separating male pupae:

1. Partition the contents of each $3000 \mathrm{~mL}$ beaker generated in step 2.1 into multiple 1900 $\mathrm{mL}$ plastic containers to reduce spillage and overburdening the separator. Prepare the plate separator by placing a rigid shallow $4000 \mathrm{~mL}$ collection container below the sluice at the base of the separator (Figure 1). Fill two $3000 \mathrm{~mL}$ graduated plastic beakers about $3 / 4$ full of tap water.

NOTE: Use a sink hose as an alternative to the $3000 \mathrm{~mL}$ plastic graduated beakers. Otherwise, beakers will need to be continuously refilled throughout the separation process. Additional details for operating the pupal separator may be found in references ${ }^{12,20}$.

2. Pour water through the space between the glass plates and adjust the top and bottom knobs clockwise or counterclockwise to allow water to continuously flow through while simultaneously generating standing water to a height of approximately $1.25 \mathrm{~cm}$ from the base of the plates. Mark the starting positions of the bottom knobs with tape. Once standing water is evenly distributed across the base of the glass plates with equal height and drain rate, begin pouring the contents of the containers of pupae and larvae through the space between the plates.

NOTE: A clear separation should be present between small (male) and large (female) pupae; otherwise, flush this batch through, and adjust the upper knobs to reduce the space between the plates.

3. While slowly pouring water through the separator, continuously rotate the bottom knobs as a pair in a counterclockwise manner $\sim 1-2$ $\mathrm{cm}$ from the tape-marked starting position until most or all larvae have washed through and slid down the sluice into the collection container.

NOTE: As one hand is being used to pour water, the other hand rotates the knobs one at a time, but equally and in small increments, to slowly open the plates. Most larvae are quickly washed through, but there will be some larvae caught with the male pupae. These straggler larvae will be irradiated but will lag in development and not eclose to adults with the focal cohort of pupae.

4. Discard or recycle the larvae back into the colony, but in either case, remove them from the collection container before the male pupae begin washing through the separator. Pause the process by ceasing the water flow while the collection container at the base of the sluice is cleared of larvae by pouring through a \#30 sieve, which is backwashed into a separate container.

NOTE: Larvae flow through first, followed by male pupae, and finally the females (Figure 1).

5. Continue to pour water and rotate the bottom knobs until the male larvae are washed through and separated into the collection container. Pause the process to check for and remove larvae from the collection container before transfer of the male pupae in the next step. 
NOTE: The number of flushes needed to separate the males depends on the tempo of the water pour and the speed at which the knobs are rotated. It usually takes $2000-2500 \mathrm{~mL}$ of water to flush the larvae out, $1000-1500 \mathrm{~mL}$ to flush out the male pupae, and $200-400 \mathrm{~mL}$ to flush out the female pupae.

6. Pour the male pupae out of the collection container through a \#20 sieve over a sink. Use a $1000 \mathrm{~mL}$ squeeze bottle of tap water to backwash the male pupae from the sieve while pouring into a separate $1900 \mathrm{~mL}$ container.

7. Once all the male pupae have been separated, continue to pour water through the separator, and adjust the lower knobs to flush through the female pupae. Transfer the females using the sieve process described in step 2.2.1.6 into a separate container until all immature mosquitoes are flushed out of the separator. Discard the female pupae. Once the batch has been processed, return the knobs to their original starting positions, and repeat the process with the next batch. Once all the batches are processed, leave the plates open so that the separator can dry.

NOTE: There is no perfect separation using this device, which requires patience and practice. Stubborn pupae or larvae can be dislodged with a heavy flow of water, but not to the extent that it pushes them to the sides, which could contaminate future inundations.

2. For two operators separating the male pupae, modify section 2.2.1 as follows.

1. First operator: Pour water through the separator, and rotate the knobs incrementally to separate larvae, male pupae, and female pupae.

2. Second operator: Once each stage is collected into the sluice container, sieve out the contents of the sluice container to partition the larvae, male pupae, and female pupae into multiple, separate sluice collection containers. Keep the large beakers filled with water if a sink hose is not available. 


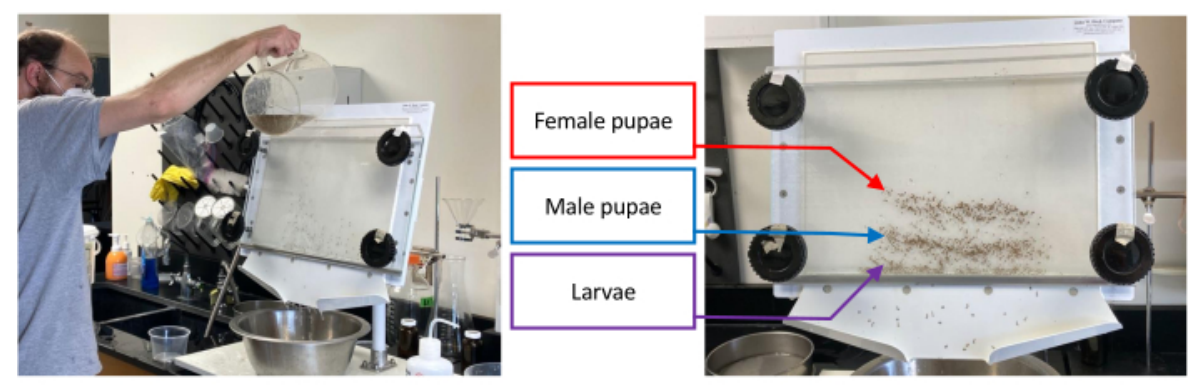

Figure 1: Pupal separator containing a batch of immature Aedes aegypti. Separation begins by pouring water through the separator while rotating the bottom knobs 1-2 cm counterclockwise until the targeted set, i.e., larvae, male pupae, or female pupae, has been isolated as much as possible from the sets that remain (left image). Right image shows separation of larvae (lowest band), male pupae (middle band), and female pupae (upper band). Please click here to view a larger version of this figure.

\section{Preparation of male Aedes aegyptipupae for irradiation}

1. Partition the male pupae into plastic $60 \mathrm{~mm}$ Petri dishes. NOTE: The number of Petri dishes needed depends on how many male pupae are available for irradiation: one larval rearing pan from step 1.2 will fill approximately 1.5 Petri dishes. The age of the pupae age ranges from 1 to $40 \mathrm{~h}$ old. In this protocol, the smaller diameter deeper half of the Petri dish is called the bottom, and the larger diameter shallower half is called the top.

1. Prepare precut disks of filter paper to fit the inside diameter of the Petri dish bottom. Place one water-moistened filter paper disk in each of the bottoms of the Petri dishes to keep the pupae hydrated throughout transportation and the irradiation process.

2. Transfer the pupae into Petri dishes. Strain the male pupae collected in step 2.2.1.6 with a sieve and wash the pupae into a $1000 \mathrm{~mL}$ graduated beaker with as little water as possible. Carefully pour the pupae into Petri dishes until each filter paper disk is evenly covered with a single layer of pupae (Figure 2A - C). Arrange the Petri dishes at the edge of a table in a row to facilitate pouring.

NOTE: An alternative to straining the pupae is to snip the tip of a plastic $3 \mathrm{~mL}$ Pasteur pipette to a diameter large enough to accommodate the pupae. Use the pipette to transfer the pupae from the container produced in step 2.2.1.6 directly on to the filter paper disks so that there is a single, closely packed layer of pupae in each Petri dish. This is only practical for small batches.

3. Use an unaltered $3 \mathrm{~mL}$ Pasteur pipette to remove standing water from the Petri dish bottom to prevent 
pupal movement during the sexing step (3.2) and

transport.

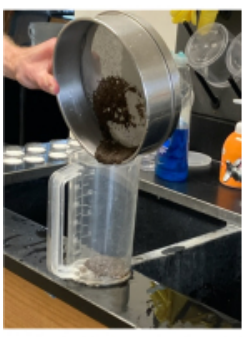

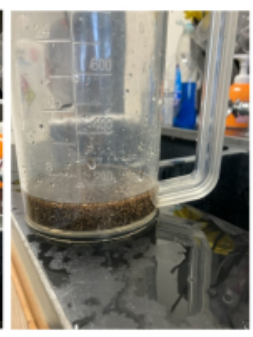

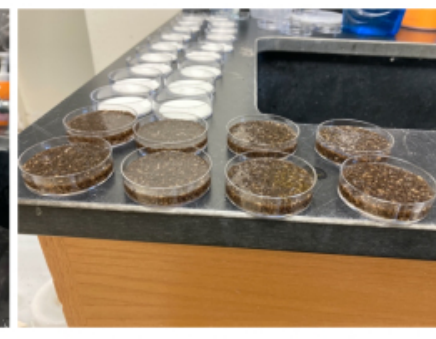

C

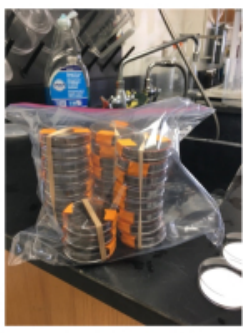

Figure 2: Transferring pupae to Petri dishes for irradiation. (A) Sieved pupae are poured and backwashed into a 1000 $\mathrm{mL}$ plastic beaker. (B) Minimal water is retained in the beaker to facilitate pouring into Petri dishes. (C) Petri dishes lined up along the edge of a surface to facilitate pouring in a single layer of pupae. (D) Petri dishes loaded with pupae are stacked and secured for delivery to the irradiation facility. Please click here to view a larger version of this figure.

2. Sex the pupae to check for contamination with females. Under a dissecting scope, use probes to rotate each pupa to check the ventral surface for a large size genital lobe (Figure 3) indicating male sex. Remove and discard the pupae with reduced or small genital lobes that indicate females and replace with an equal number of male pupae to maintain the correct count.
NOTE: In an operational program, this step is not practical because of large numbers of mosquitoes, and the fact that separation, transfer, irradiation, and preparing cages after irradiation are all done in one day with limited time. Quality-checking a sample from select Petri dishes can be done, particularly in early phases of development of the SIT program. 

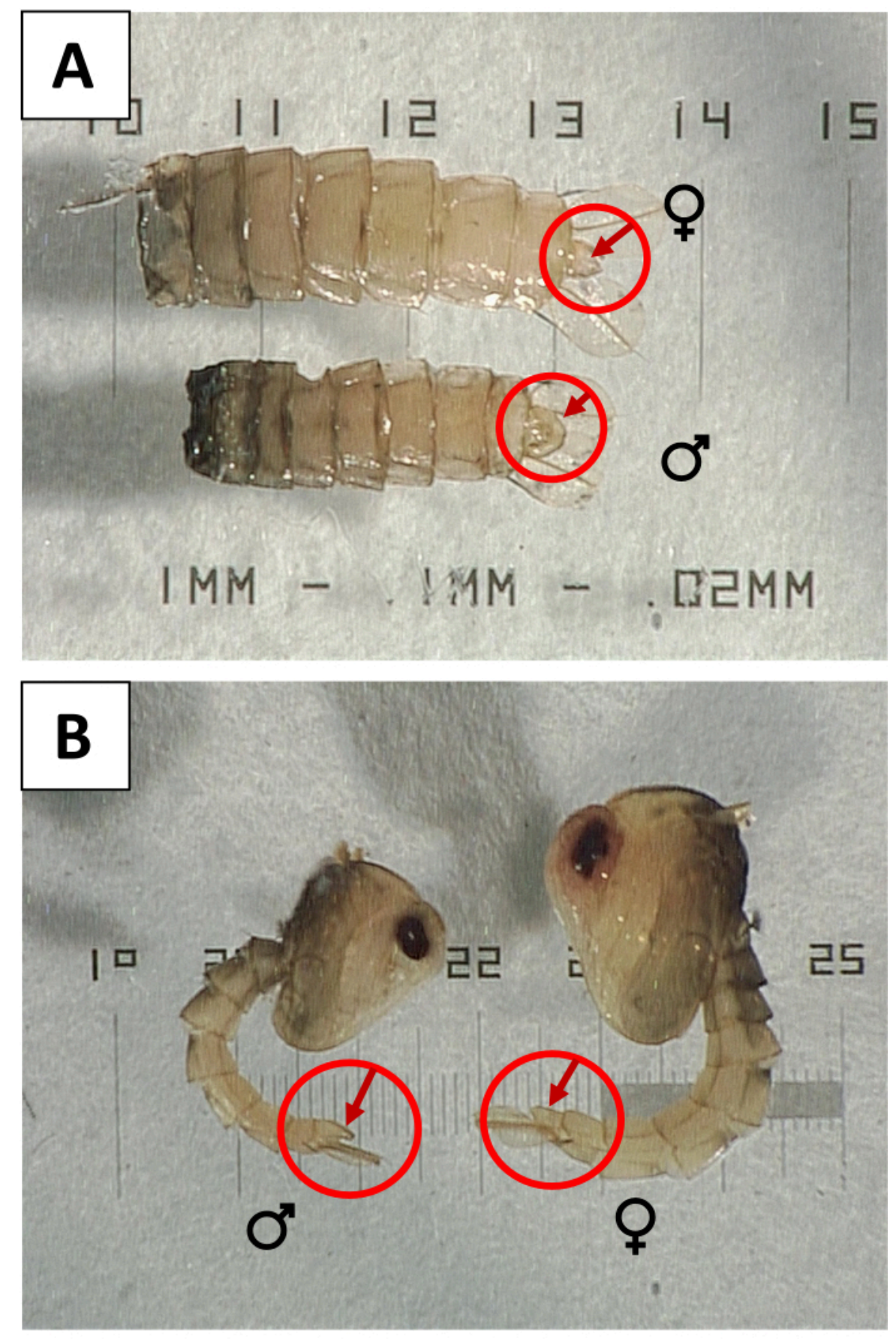

Figure 3: Sexing pupae using the genital lobe. (A) Ventral and (B) lateral views of female $(\uparrow)$ and male $(\hat{O})$ Aedes aegypti pupae, with genital lobes indicated to show the sexual dimorphism. Please click here to view a larger version of this figure.

3. Cover the bottoms with the tops of the Petri dishes, and secure with laboratory tape. Bundle the taped Petri dishes with elastic bands in stacks sized to fit in the irradiation chamber, and seal inside a labeled $3.8 \mathrm{~L}$ 
resealable bag (Figure 2D). Do not allow pupae to remain uncovered for $>1 \mathrm{~h}$.

\section{Irradiation of male Aedes aegyptipupae}

1. Prepare dosimetry film from the same lot by cutting 1 $\mathrm{cm}^{2}$ squares of films and placing each square into its individual envelope.
NOTE: All film used on each day is cut at the same time. This reduces the small amount of variation induced by storage. The number of squares needed for each stack is $1+$ (number of Petri dishes).

2. Prepare a kit to take into the irradiation facility, which should include a laboratory timer, laboratory tape, permanent marker, prepared envelopes of dosimetry film, dosimetry badge, and a laboratory note sheet with checklists to keep track of key information (Figure 4). 


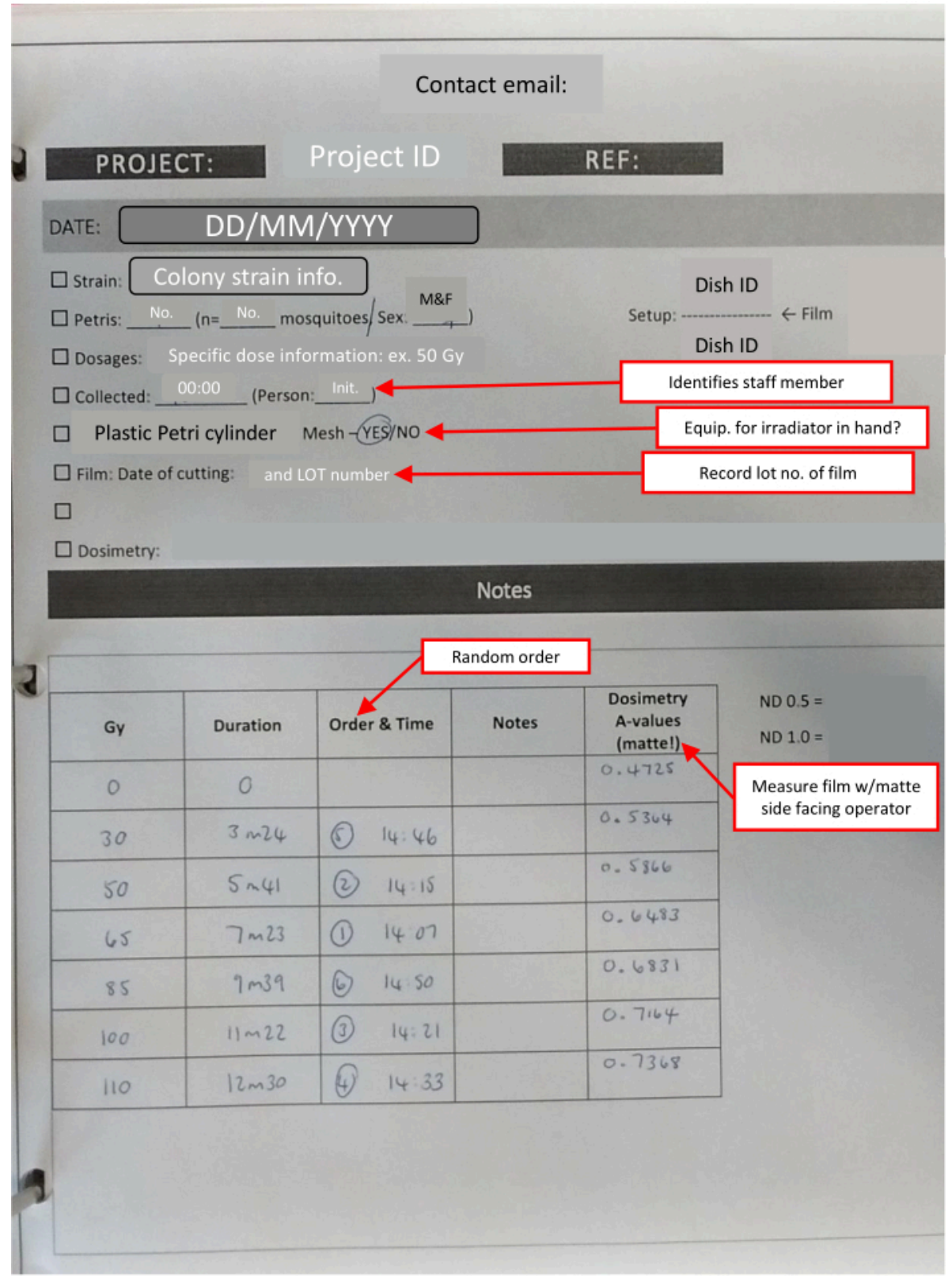

Figure 4: Laboratory book outline-IR sheet completed for a dose response set. Text boxes outlined in red (marked by red arrows) indicate useful notes on the different sections and reiterate key information. Please click here to view a larger version of this figure.

3. Transport the pupae to the irradiation facility. Place the Petri dishes of male pupae from step 3.3 in insulated containers and store out of direct sunlight and with air conditioning during transport. 
4. Prepare stacks of Petri dishes for irradiation at the irradiator. Stack the appropriate number of Petri dishes to fit in the irradiation chamber with a dosimetry film envelope centered between each dish and at the top and bottom of the stack. Secure the envelopes and the whole stack with laboratory tape to prevent spills and facilitate placement of the stack in the chamber.

5. Irradiate the Petri dishes of male pupae. Place the stack of Petri dishes on the rigid metal mesh in the chamber to position at the correct height for the optimal cone of exposure based on previous dose-mapping of the specific irradiation unit ${ }^{21}$. Activate the turntable on the irradiator and irradiate, simultaneously initiating the laboratory timer. Irradiate for the appropriate interval to accomplish the desired dose (examples are shown in Table 2).

NOTE: This protocol is based on a cesium-137 irradiator (see the Table of Materials) and a target dose of 50 Gy. Because Cs-137 decays over time, the dose rate is adjusted every year by performing a dose-response series using alanine dosimeters, supplemented with radiochromic film for routine dosimetry and alanine in approximately $10 \%$ of irradiated samples. Given the current dose rate of $8.8 \mathrm{~Gy} / \mathrm{min}$, achieving the target dose of $50 \mathrm{~Gy}$ requires $5 \mathrm{~min}, 41 \mathrm{~s}$ of exposure. Routine film dosimetry occurs as described in step 4.7. Alanine pellet dosimetry is performed at either the National Center for Electron Beam Research at Texas A\&M University or at the National Institute of Standards and Technology in Gaithersburg, MD, USA.

\begin{tabular}{|c|c|}
\hline Dosage $(\mathrm{Gy})$ & Time (based on 8.8 Gy/min) \\
\hline 0 & $\mathrm{NA}$ \\
\hline 10 & $1 \mathrm{~min} 8 \mathrm{~s}$ \\
\hline 30 & $3 \min 24 \mathrm{~s}$ \\
\hline 50 & $5 \min 41 \mathrm{~s}$ \\
\hline 65 & $7 \min 23 \mathrm{~s}$ \\
\hline 85 & $9 \min 39 \mathrm{~s}$ \\
\hline 100 & $11 \min 22 \mathrm{~s}$ \\
\hline 110 & $12 \min 30 \mathrm{~s}$ \\
\hline
\end{tabular}

Table 2: Example dosage times for the Cesium-137 irradiator.

6. Once the prescribed time has elapsed, remove the Petri dishes from the irradiator, and carefully dismantle the stack. Label all the Petri dishes and film envelopes with the date and location in the stack. Seal the envelopes and store for dosimetry. Repack the Petri dishes into the insulated container for transport back to the main laboratory.

NOTE: Record whether adults emerged during irradiation and mark the affected Petri dish so that the adult(s) will 
not escape when the pupae are set into the rearing cages (step 5.2).

7. Confirm the irradiation dose with dosimetry film by measuring the film approximately $24 \mathrm{~h}$ post exposure. Activate the dosimetry reader and allow it to equilibrate to room temperature. Load the film using forceps supplied with the reader and follow the manufacturer's instructions for reading the irradiated film as well as the unirradiated blank film from the same batch. Zero the reader with no film between readings, and record data as in the example data sheet shown in Figure $\mathbf{5}$.

NOTE: This protocol is based on ND0.5 and ND1.0 QA

Filter Set standards. It is important to measure the film with the matte side facing the operator.

\begin{tabular}{|c|c|c|c|c|c|c|c|}
\hline & & & $\begin{array}{l}\text { Location } \\
\text { of film } \\
\text { when } \\
\text { petri } \\
\text { dishes are } \\
\text { stacked: } \\
\text { e.g. top, } \\
\text { middle, } \\
\text { bottom }\end{array}$ & & $\begin{array}{c}\text { Amber }(\mathrm{A}) \\
\text { value }\end{array}$ & & \\
\hline Date_dosimetry & Date_irradiation & Date_film cut & Location & Target dose (Gy) & $O D(590 \mathrm{~nm})$ & Temp & Notes \\
\hline 20-Nov & & $20-$ Oct-16 & & ND 0.5 & 0.538 & 25.7 & Example data \\
\hline 20-Nov & & 20-Oct-16 & & ND 1.0 & 0.969 & 25.6 & Example data \\
\hline 20-Nov & 20-Oct-16 & 20-Oct-16 & & 0 & 0.3215 & 25.8 & Example data \\
\hline 20-Nov & $20-0 c t-16$ & $20-0 c t-16$ & & 30 & 0.4244 & 25.8 & Example data \\
\hline 20-Nov & 20-Oct-16 & 20-Oct-16 & & 50 & 0.4766 & 25.9 & Example data \\
\hline 20-Nov & 20-Oct-16 & $20-$ Oct-16 & & 65 & 0.5232 & 25.9 & Example data \\
\hline 20-Nov & 20-Oct-16 & $20-$ Oct-16 & & 85 & 0.565 & 25.9 & Example data \\
\hline 20-Nov & $20-$ Oct-16 & $20-$ Oct-16 & & 100 & 0.5928 & 25.9 & Example data \\
\hline 11-Aug-17 & 10-Aug-17 & 02-Aug-17 & Top & 60 & 0.5275 & 27 & Example data \\
\hline 11-Aug-17 & 10-Aug-17 & 02-Aug-17 & Middle & 60 & 0.5318 & 27 & Example data \\
\hline 11-Aug-17 & 10-Aug-17 & 02-Aug-17 & Bottom & 60 & 0.5227 & 27 & Example data \\
\hline
\end{tabular}

Figure 5: Dosimetry data sheet populated with example data. The column headings prompt the operator to capture key data for later analysis. Please click here to view a larger version of this figure.

\section{Rearing of irradiated male Aedes aegyptipupae into adults}

1. Clean and prepare small plastic $30 \mathrm{~cm} \times 30 \mathrm{~cm} \times 30$ $\mathrm{cm}$ rearing cages so that they are ready for irradiated pupae upon return to the main laboratory. For every 2 Petri dishes of irradiated male pupae, prepare 1 rearing cage. Stock each rearing cage with a half-filled plastic cup containing $460 \mathrm{~mL}$ of tap water and one $10 \%$ sucrose solution container, as described in step 1.1.3.

2. Immediately transfer the irradiated pupae to the prepared rearing cages after returning from the irradiation facility. Use a squeeze bottle of water to carefully wash the pupae from each Petri dish into the $460 \mathrm{~mL}$ of water in the plastic cup in each rearing cage. After $24 \mathrm{~h}$, transfer the cups to new, clean rearing cages containing the nutrition source, 
and await remaining eclosion of adult, male irradiated Aedes aegypti.

NOTE: If pupae have emerged during the irradiation process, open the Petri dishes with the fliers into an empty cage, and then continue with step 5.2. Discard the males collected in this cage. Pupae are transferred to new rearing cages after $24 \mathrm{~h}$ because males emerge before females of the same cohort and isolating the days of emergence can reduce the incidence of female contamination and ensure accurate aging of males.

\section{Marking and weighing irradiated adultAedes aegypti males}

NOTE: This section of the protocol assumes two people are conducting the tasks; for 1 person, see 6.4.
A

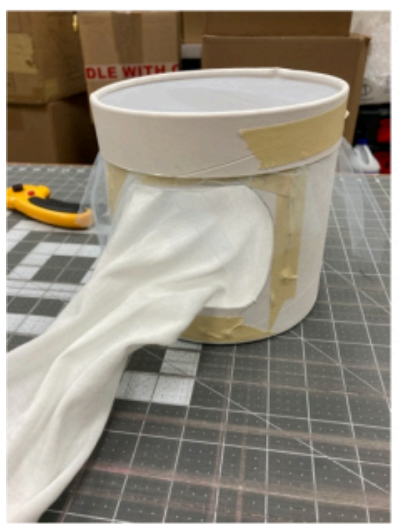

B

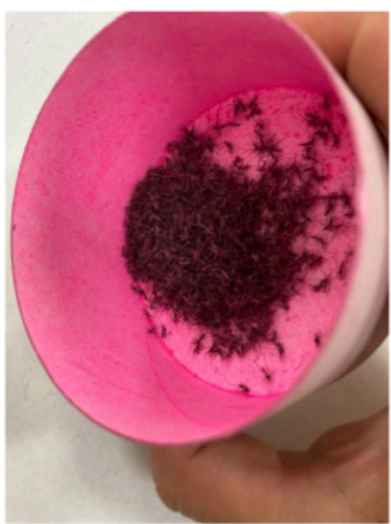

C

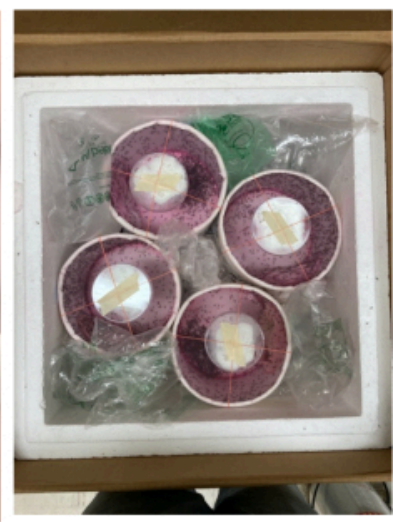

Figure 6: Packing marked, irradiated, male Aedes aegypti into release containers. (A) Release container showing stockinette fastened to a hole cut in the side of the cardstock cylinder with masking tape, staples, and hot glue. The bezel is in place with a masking tape label backing affixed to the side. The bezel is retaining the tightly pulled tulle mesh cover; an elastic band (not visible) is also holding the tulle in place under the bezel. (B) Batch of anesthetized males in the process of being tumbled in pink dye in a small cardstock cup. (C) Four release containers inside insulated shipping container. Note the stockinette sleeves are oriented to the middle of the shipping container, packing materials are tucked around the release containers, and nutrition and hydration sources are in place on top of each release container covered by an inverted Petri dish bottom held fast by crossed elastic bands and bits of tape. Please click here to view a larger version of this figure.

1. Prepare cardstock release containers.

1. Cut an $11.5 \mathrm{~cm}$ diameter hole in the side of a lidded, cylindrical $3.9 \mathrm{~L}$ cardstock container at $1.5-3.0 \mathrm{~cm}$ from the bottom so that the hole will not be covered by the lid (Figure 6A).

2. Cut a $40-50 \mathrm{~cm}$ length of stockinette, and staple one end of it around the inside of the $11.5 \mathrm{~cm}$ hole cut 
in 6.1.1. Use a standard office stapler opened up so that staples can be forced through the stockinette and the cardstock from the inside of the container and into a suitable work surface such as a block of hard extruded polystyrene foam. Crimp the staples using a flat-headed screwdriver to tightly secure the stockinette to the perimeter of the hole and fasten masking tape over the crimped side to prevent snags. Seal the edge of the stockinette on the inside of the cylinder to the cardstock with hot glue and cross-check the entire assembly for escape holes.

3. Create a retaining bezel by removing the inner disk from the lid and cut a $33 \mathrm{~cm} \times 33 \mathrm{~cm}$ square of nylon tulle mesh fine enough to retain adult male Aedes aegypti mosquitoes. Place the mesh on the open end of the cylinder and fit the bezel over the mesh to hold it in place with no gaps. Pull the mesh downward so that it protrudes below the bezel to make the mesh taught across the open end and seal the protruding mesh tight against the cylinder with a rubber band.
4. Place a $10 \mathrm{~cm}$ piece of masking tape on the side of the bezel covered with an $8 \mathrm{~cm}$ piece of labeling tape so that the labeling tape can be replaced easily without tearing the bezel.

NOTE: Release containers are durable and can be reused $>10 x$ with proper handling. Before introducing each new batch of marked, irradiated, adult, male mosquitoes, check that the stockinette is securely attached to the container, and promptly make repairs if needed.

2. Prepare the weighing and marking station.

1. Pour approximately $50 \mathrm{mg}$ of marking dye into a 240 $\mathrm{mL}$ cardstock container, and spread the dye out as a coat of powder evenly around the inner surfaces of the container. Gently tap to discard excess dye. Use clearly labeled cups for multiple dyes to keep the colors separated.

2. Tare a $100-500 \mathrm{~g}$ weighing boat on a $0.0001 \mathrm{~g}$ electronic balance; create a data form (Table 3); and tape together four sheets of $215.9 \mathrm{~mm} \times 355.6 \mathrm{~mm}$ copy paper to make a $431.8 \mathrm{~mm} \times 711.2 \mathrm{~mm}$ work surface.

\begin{tabular}{|c|c|c|c|c|c|c|}
\hline $\begin{array}{c}\text { Release } \\
\text { Container }\end{array}$ & $\begin{array}{c}\text { Weight of } \\
\text { Mosquitoes }\end{array}$ & Cage Number & $\begin{array}{c}\text { Females } \\
\text { in Batch }\end{array}$ & $\begin{array}{c}\text { Number } \\
\text { of Males }\end{array}$ & $\begin{array}{c}\text { Release } \\
\text { Container }\end{array}$ & Total Mass \\
\hline PINK I & 0.024 & D1 \#1 & & 25 & PINK I & 2.03 \\
\hline PINK I & 2.007 & D1\#1 & 7 & & PINK II & 1.99 \\
\hline PINK II & 1.990 & D1\#1 & & & PINK III & 2.03 \\
\hline PINK III & 0.026 & D1 \#3 & & & & \\
\hline PINK III & 2.000 & D1 \#3 & 18 & & & \\
\hline
\end{tabular}

Table 3: Weighing station data table. 
3. Mark quantified batches of adult irradiated males with fluorescent pigment.

1. Transfer adult mosquitoes (2.5-3.5 days old) from each rearing cage into aspirator vials. Remove nutrition sources from the rearing cage from step 5.2 and place the entire cage into a large $\mathrm{CO}_{2}$ chamber for 5-7 min, knocking the sides of the container to dislodge mosquitoes that may be clinging to the rearing cage. After the exposure time has elapsed, remove the rearing cage from the chamber, and aspirate all the adult mosquitoes into a series of plastic aspiration vials.

NOTE: Administration of the $99.5-100 \% \mathrm{CO}_{2}$ is from a tank with a regulator, brass barb, and silicone tubing channeled into the chamber at a flow rate of $6 \mathrm{~L} / \mathrm{min}$. The number of vials needed to clear the rearing cage depends on how many mosquitoes are in the cage and the proficiency of the operator, but 3-5 vials are typically required per cage. Choose an aspirator that has small quick-change vials to facilitate the management of adult mosquitoes in batches, for example, one using a $60 \mathrm{~mL}$ polystyrene collecting vial sealed with $20 \times 20$ mesh aluminum screen on one end and a clear acetate flap valve on the other. The entire cage is anesthetized before aspiration to reduce time to transfer mosquitoes to vials to reduce the stress on mosquitoes and keep the protocol tractable.

2. Sort all adult mosquitoes from each rearing cage by sex.

1. Expose the first vial from step 6.3.1 to $\mathrm{CO}_{2}$ in a small chamber for $4 \mathrm{~min}$, and then gently shake the anesthetized mosquitoes out, spreading them across the white paper work surface prepared in step 6.2.2.

2. Load a new empty vial into the aspirator, and carefully aspirate all males from the work surface, and pass this vial to the weighing station (step 6.3.3.). Tally any females left behind, and aspirate them into a separate vial and discard, along with any crushed males.

3. Repeat this process with the remaining vials from 6.3.1, but at some point in the sex-sorting process, generate a separate vial with only 25 males that is also passed to the weighing station. Repeat step 6.3.2. for each rearing cage.

NOTE: While processing irradiated adult males for sexing, weighing, and marking, keep track of the number of females in each batch, which are key data for troubleshooting and quality assurance of pupal sex sorting and the entire process. If the female count is higher than expected, a second person should extract females while the main operator aspirates the males. It is important to weigh a sample of 25 males from each rearing cage to calculate an average weight per mosquito, which will be used to estimate the numbers of marked irradiated males released at the end of the protocol.

3. Weigh and dye batches of adult male mosquitoes.

1. At the weighing station, place the first vial of adult male mosquitoes from the sexing station (section 6.3.2) into a small $\mathrm{CO}_{2}$ chamber for 2 min, and carefully shake the mosquitoes into 
the tared weighing boat prepared in step 6.2.2. Record the weight of the mosquitoes and pour the mosquitoes into the dye cup prepared in 6.2.1.

2. Slowly tilt and rotate the cup 1 full rotation clockwise and counterclockwise so that the mosquitoes contact the powder coating on the inner surfaces of the cup and are all lightly dusted with dye (Figure 6B). Pour the marked mosquitoes into a weighing boat.

3. Proceed quickly to the next step so that the mosquitoes do not recover and escape. Repeat this step until all vials from section 6.3.2 have been processed.

NOTE: The weight of the males from the separate vial of 25 males generated for each rearing cage in section 6.3.2 is recorded and used to calculate the mean weight per male from that cage.

4. Load the release containers with marked irradiated adult males. Slightly fold the weighing boat containing anesthetized, marked, irradiated, male mosquitoes from the end of section 6.3.3. to create a channel, and then direct this channel through the stockinette sleeve to transfer the males into the release container. Continue to add mosquitoes until approximately $2.0 \mathrm{~g}$ or $1500-3000$ male mosquitoes are in the release container and tie the stockinette sleeve closed. Mark the labeling tape on the bezel of the release container with dye color, container number, and total weight of mosquitoes, and copy these data to the form from step 6.2.2.

NOTE: Handling of mosquitoes at any life stage induces stress and can reduce survival or vigor.
The series of anesthetizations described in this protocol could impact the mosquitoes; however, attempts to pursue and aspirate non-anesthetized mosquitoes at each step would induce greater stress and an unsustainable protocol. Divide the total weight of mosquitoes in each release container by the average weight per male mosquito generated in section 6.3.3 to derive an estimate of the number of males in that release container; each release container should have no more than $2 \mathrm{~g}$ of males, which equates to approximately 1 large rearing cage.

4. Modifications to the marking protocol for a single operator

1. Conduct sex sorting for all large cages first. Expose each large cage to $\mathrm{CO}_{2}$ for $4-5 \mathrm{~min}$ and aspirate all the mosquitoes into 4-5 vials. Expose each vial to $\mathrm{CO}_{2}$ for 2-3 min, tip out all the mosquitoes on to the white paper work surface, remove the females and tally, and return the males to their large population cage.

\section{Weighing and marking}

1. Start with the first male holding cage produced in step 6.4.1: remove the nutrition source and anesthetize the males in a large $\mathrm{CO}_{2}$ chamber for 5-7 min. Aspirate the anesthetized males evenly into separate vials. Repeat this step with each holding cage in the order they were produced.

NOTE: Approximately 2-3 crowded vials will be produced per holding cage. Processing male holding cages in the order they were produced maximizes recovery time for each cage of males. 
2. Anesthetize the first vial produced in step 6.4.2.1. for $1-2$ min in a small $\mathrm{CO}_{2}$ chamber. Pour out a small number of mosquitoes onto the white paper work surface, aspirate 25 male mosquitoes into a new vial, and process as in 6.3.2 to determine the mean weight per male for that cage. Return any additional males to the source vial, or aspirate into a new separate vial to be processed later; proceed with weighing, marking, and transfer to the release containers for the rest of the males in the first vial as described in the rest of step 6.3. Repeat step 6.4.2.2. (except for isolating 25 males into a separate vial) for the rest of the vials produced in step 6.4.2.1. in the order they were produced, and then move on to the next sex-sorted rearing cage produced in 6.4.1.

NOTE: Workflows with one person are slower, and some male mosquitoes will need to be anesthetized multiple times. Constantly search for and remove female mosquitoes.

\section{Packing and shipping release containers of marked, irradiated, adult male Aedes aegypti}

1. Prepare the release containers for shipment. Once a release container is filled with marked males, place 4 cotton balls dampened with $10 \%$ sucrose solution on the mesh lid and cover with an inverted bottom of a Petri dish held in place by two rubber bands stretched around the entire container and over the Petri dish to form a cross. Place a piece of masking tape over the $X$ of the two rubber bands to keep them in place on top of the inverted Petri dish.
NOTE: Ensure the cotton balls with $10 \%$ sucrose solution are not saturated to the point of dripping, which will damage the container and trap and kill mosquitoes.

2. Pack cardstock release containers into an extruded polystyrene foam shipping cooler. Poke 4 ventilation holes through the cooler lid and occlude with cotton to keep out ants and keep in escaped mosquitoes. Place 4 release containers upright into the shipping cooler with the stockinette of each container facing the center (Figure 6C). Tuck bubble wrap between each container and in the center to stabilize them. Fill in the rest of the space in the shipping cooler with air pillows or stack a second layer of 4 release containers directly on top of the first layer and similarly stabilize with air pillows.

NOTE: The release containers should be sufficiently stabilized to not move when shaken. The temperature inside the package is ambient.

3. Prepare the shipping cooler for delivery. Seal the shipping cooler with the ventilated lid, and place into the cardboard overpack, tape shut, and ship via overnight express to the release location.

\section{Representative Results}

Vigilant and adequate mosquito rearing consists of wellbalanced availability of males and females in colony cages, maintenance of fresh sucrose solution and honey, and consistent high-quality blood feeding. These conditions will provide for densely packed egg sheets optimal for use in SIT larval rearing pans. Proper storage and usage of dried egg sheets, such as systematic labeling to facilitate use from oldest to newest, will support uniform hatching across all pans. Filling all larval rearing pans with water prior to hatching can diminish the time for which the egg sheets are in hatch containers and promote healthy development. 
Maintenance of larval pans from hatch to pupation requires careful engagement by colony personnel as some pans may need more or less food or additional water depending on development stages and environmental variables. If there are issues with the stage of development by the scheduled day of pupal sex separation, adjustments should be made earlier in the process such as hatching earlier or later, adjusting food, or changing the incubator temperature.

The rearing process in this protocol does not render all eggs hatched in time to develop into pupae that can be irradiated and used for control purposes. Between 20 and $50 \%$ of the colony-reared mosquitoes will still be larvae by the time the pupae need to be separated. However, these larvae are not squandered, but allowed to mature for $24 \mathrm{~h}$ to render additional pupae that can be combined with female pupae from the previous day's separation and recycled back into colony cages. In the colony cages, pupae will be allowed to mature into adults, mate, bloodfeed, and produce eggs that sustain the SIT project.

Separating pupae, pouring pupae into Petri dishes, irradiation, and placement into adult holding cages after irradiation must happen in one day; hence, adequate time should be allotted to process all steps comfortably. The assembly and preparation of release containers should be done prior to the marking process. When shipping boxes are returned from the release site, release containers should be inspected and prepared for their next use. Discarding wet cotton balls, airing out wet release containers, cleaning Petri dishes, replacing mesh, and removing elastic bands from the container, while not in use, will greatly prolong the life of the release containers.

Given the worldwide reality of the COVID-19 viral pandemic, this protocol that is typically a multi-person operation has been modified to be tractable by one person working alone in a lab for each step. The steps in the process that are hindered most by a one-person scenario are the sexing, marking, weighing, and colony-rearing maintenance steps. Separating pupae by sex by one person should be sufficient if there are multiple separators operating simultaneously in different rooms. In a pandemic situation wherein social distancing occurs in the workplace, equipping multiple stations is required to complete steps from sexing to packing. Depending on the speed of the operator, it takes one person $\sim 4 \mathrm{~h}$ to sex 15,000 mosquitoes and then another $1-2 \mathrm{~h}$ to mark, weigh, and package them. A two-person scenario diminishes the time during which mosquitoes are anesthetized for marking and reduces the overall work time. Yet, even in a two-person scenario, allocating the full $2.0 \mathrm{~g}$ of mosquitoes per release cage can be challenging due to limited work time while the mosquitoes are sedated. Although the process of cleaning and preparing larval and adult rearing materials is extremely time-consuming and labor-intensive, it can be partitioned such that individual operators can work independently and safely during a pandemic.

Releasing adult, marked, irradiated Aedes aegypti males is outside the scope of this protocol but is presented here in brief. The process of releasing marked, irradiated, male mosquitoes starts by determining a uniform release distribution of the release containers based on weights (and thus, inferred numbers of sterile males), as reported in Table 3. After shipments are delivered to the vector control district, the boxes are opened, and the release containers evaluated for any issues with mortality or condition of the release containers. Mosquitoes in the release containers are then allowed to acclimate to ambient temperature and humidity for 1-2 $\mathrm{h}$ prior to transport to the treatment area. Release sites in the treatment area are identified after intensive surveillance 
for hot spots of wild populations of Aedes aegypti. The timing, frequency, and density of releases is balanced by the bionomics of the species as well as meteorology, public support, and laboratory-rearing capabilities.

As specific release containers are matched to particular release sites, the label must be cross-checked before the release container is opened by cutting the mesh on the top, allowing the operator to deform the mesh so that a portion of the males may escape. This fractional release method is repeated at each assigned release point for the container until all freely flying males have been released. This process is then repeated for each release container at their respective assigned release location until all containers have been processed. Optionally, after the mosquitoes have been released, any dead or disabled mosquitoes that did not leave freely can be collected into Petri dishes and labeled to be counted by hand or weighed to correct the estimated number released. Ongoing and pervasive surveillance of adult, egg, and immature stages of wild Aedes aegypti in the target area, and possibly in non-intervention control sites, is conducted to assess the efficacy of the SIT operation.

\section{Discussion}

Initiation of a control program featuring SIT that uses radiation requires the establishment of a local strain of Aedes aegypti. This step is critical and can allow SIT to truly distinguish itself from similar control technologies. By developing the project from a local strain of mosquito, the males generated will likely have behaviors that allow them to adapt to environmental changes and cues and to locate and mate with wild females in the vicinity. Furthermore, the release of irradiated local males may not generate negative public opinion compared to, say, release of a non-local strain of genetically modified mosquitoes that could, for example, introduce novel alleles into the local mosquito population.

Expending substantial resources to rear vast quantities of mosquitoes only to be able to use about half of them for control purposes is a limitation of the Aedes aegypti SIT program. Refinements should be made to the rearing protocol to condense the maturation of larvae into more defined timeframes when the pupae will be ready. This would allow more pupae to be collected at the optimal time of separation. However, additional pupae to process increases the risk of more females pupating when the pupae are collected and therefore increasing the likelihood of females ending up in Petri dishes with males and possibly being released. Although the lifespan, bloodfeeding behavior, and oviposition behavior in irradiated female Aedes aegypti pupae are reduced in adults, it is not a good strategy to release females incidentally alongside irradiated males ${ }^{22}$. Therefore, it should remain a priority to minimize the number of females inadvertently separated, irradiated, marked, and released with males.

Success of an SIT program ultimately relies on successful mate competition by colony-reared, irradiated males. Preserving male competitiveness relies on exhaustive experimentally derived selection of the dose and maximizing the estimated ratio of sterile:wild males in the population. Dose selection is determined by several key factors that include longevity, fertility, fecundity, and pupal mortality. It has been observed that male mosquitoes will exhibit an asymptotic fertility curve that approaches zero as radiation increases (KJL, RLA, SCB unpublished data). Simultaneously, male mosquito longevity and activity levels diminish exponentially as the radiation dose increases (KJL, RLA, SCB unpublished data). Therefore, rather than identifying a dose that yields $99.9 \%$ sterility in males, it 
is preferable to focus on a lower sterility percentage while supporting survivorship. Once a dose range is identified that does not differentiate longevity or pupal mortality of irradiated males from that of non-irradiated males, additional assessments on fertility should be conducted to identify a dose that renders males overwhelmingly sterile, yet competitive.

Simultaneously, it is critical to compare the number of male mosquitoes in the population to that of released irradiated males. This can be accomplished by collecting males from various locations in and around the target release area repeatedly from the same location and before, during, and after initiation of the SIT program. A mark, release, recapture study should be conducted to assess the ratio of wild male mosquitoes to released mosquitoes. A mark, release, recapture study relies on the release of a known number of marked mosquitoes from a specific point and their later recapture at points in close proximity surrounding the initial release point. By comparing the number of recaptured males and wild males at distances from the release point, it is possible to estimate the general wild population of males in the area so that competitive ratios of sterile males can be released $^{23}$. Maximizing the ratio of sterile:wild males can be achieved by releasing more sterile males and/or by reducing the wild population by classical control means such as source reduction, immature control, or adulticide treatments.

To gauge the effectiveness of sterile male releases, adult collections can be compared chronologically against a nonintervention area. As sterile males are released and the number of collected males and females in an area diminishes in relation to a comparable non-intervention area, then it may be hypothesized that it is due to the released sterile males successfully outcompeting local fertile males. This effect can also be observed in oviposition trap cups deployed in both the intervention and non-intervention sites. Eggs may still be produced in the intervention site, but if fewer hatch than those from the non-intervention site, it may be hypothesized they are not fertilized because of females mating with sterile males. More and more oviposition of unfertilized eggs could eventually lead to reduced oviposition due to nonreplacement of females in the intervention site ${ }^{8,24}$.

Future directions of SIT technology and programs naturally expand into additional medically important mosquito species. For instance, this technology may be readily adapted to control Aedes albopictus, given the very similar bionomics of Aedes aegypti and Aedes albopictus. Other disease vector mosquito species of interest include Culex quinquefasciatus, Culex tarsalis, and various Anopheles species. Improving the efficacy of this technology depends on increasing the capacity of male pupae produced at a given time, which could be achieved through genetic manipulation or artificial selection, and improving male competitiveness, which could be achieved by increasing virility, fertility, or longevity.

Ultimately, SIT programs are not a silver bullet for controlling mosquitoes. They are instead a tool in a suite of other control techniques, such as IVM programs, that cross-compensate for weaknesses among techniques. For example, whereas chemical control offers rapid and cheap control, it also fosters the development of resistance and non-target mortality; and whereas SIT is species-specific and is not likely to generate resistance, SIT males must be produced and released in perpetuity to control immigrating populations from outside the vector control district.

\section{Disclosures}

All authors have declared no conflicts of interest. 


\section{Acknowledgments}

We thank Drs. R.-D. Xue, C. Bibbs, W. Qualls, and V. Aryaprema of the Anastasia Mosquito Control District, St. Augustine, Florida, for partnership in developing the SIT program and expert insight on effective operational release of sterile male Aedes aegypti. This research was supported by the USDA-ARS and the Florida Department of Agriculture and Consumer Services (FDACS). Mention of trade names or commercial products in this publication is solely for the purpose of providing specific information and does not imply recommendation or endorsement by USDA or FDACS.

\section{References}

1. Moyes, C. L. et al. Contemporary status of insecticide resistance in the major Aedes vectors of arboviruses infecting humans. PLoS Neglected Tropical Diseases. 11 (7), e0005625 (2017).

2. Baldacchino, F. et al. Control methods against invasive Aedes mosquitoes in Europe: a review. Pest Management Science. 71 (11), 1471-1485 (2015).

3. Burkett, D. A., Cope, S. E., Strickman, D. A., White, G. B. The Deployed Warfighter Protection (DWFP) Research Program: Developing new public health pesticides, application technologies, and repellent systems. Journal of Integrated Pest Management. 4 (2), A1-A7 (2013).

4. Harwood, J. F. et al. Controlling Aedes aegypti in cryptic environments with manually carried ultra-low volume and mist blower pesticide applications. Journal of the American Mosquito Control Association. 32 (3), 217-223 (2016).

5. Morrison, A. C., Zielinski-Gutierrez, E., Scott T. W., Rosenberg, R. Defining challenges and proposing solutions for control of the virus vector Aedes aegypti. PLoS Medicine. 5 (3), e68 (2008).

6. Klassen, W., Curtis, C.F. History of the sterile insect technique. pp. 3-36. In Dyck, V.A., Hendrichs, J., Robinson, A.S. (eds.). Sterile insect technique: principles and practice in area-wide integrated pest management. Springer Netherlands, Dordrecht (2005).

7. Alphey, L. et al. Sterile-insect methods for control of mosquito-borne diseases: an analysis. Vector Borne and Zoonotic Diseases. 10 (3), 295-311 (2010).

8. Dame, D. A., Curtis, C. F., Benedict, M. Q., Robinson, A. S., Knols, B. G. Historical applications of induced sterilisation in field populations of mosquitoes. Malaria Journal. 8 (Suppl 2), S2 (2009).

9. FAO/IAEA. Guidelines for colonization of Aedes. mosquito species. Version 1.0. H. Maiga et al. (eds.). Vienna, Austria. <http://www-naweb.iaea.org/nafa/ipc/public/ Guidelines-for-colonisation-of-Aedes-mosquito-speciesv1.0.final.pdf> (2018).

10. Bond, J. G. et al. Optimization of irradiation dose to Aedes aegypti and Ae. albopictus in a sterile insect technique program. PloS One. 14 (2), e0212520 (2019).

11. Bourtzis, K., Lees, R. S., Hendrichs, J., Vreysen, M. J. B. More than one rabbit out of the hat: Radiation, transgenic and symbiont-based approaches for sustainable management of mosquito and tsetse fly populations. Acta Tropica. 157, 115-130 (2016).

12. Carvalho, D. O. et al. Mass production of genetically modified Aedes aegypti for field releases in Brazil. Journal of Visualized Experiments: JoVE. 83, e3579 (2014). 
13. Carvalho, D. O. et al. Aedes aegypti lines for combined sterile insect technique and incompatible insect technique applications: the importance of host genomic background. Entomologia experimentalis et applicata. 168 (6-7), 560-572 (2020).

14. FAO/IAEA. Guidelines for mass-rearing of Aedes. mosquito species. Version 1.0. H. Maiga et al. (eds.). Vienna, Austria. <http://www-naweb.iaea.org/nafa/ipc/public/ Guidelines-for-mass-rearingofAedesosquitoes_v1.0.pdf> (2020).

15. FAO/IAEA. Guidelines for mark-release-recapture procedures of Aedes. mosquitoes. J. Bouyer et al. (eds.), Food and Agriculture Organization of the United Nations/International Atomic Energy Agency. Vienna, Austria. <http://www-naweb.iaea.org/nafa/ipc/ public/Guidelines-for-MRR-Aedes_v1.0.pdf> (2020).

16. FAO/IAEA. Guidelines for Routine Colony Maintenance of Aedes. Mosquito Species, Version 1.0. H. Maiga et al. (eds.). Vienna, Austria. <http://www-naweb.iaea.org/nafa/ipc/public/ guidelines-for-routine-colony-maintenance-of-Aedesmosquito-species-v1.0.pdf> (2017).

17. Mamai, W. et al. Aedes aegypti larval development and pupal production in the FAO/IAEA mass-rearing rack and factors influencing sex sorting efficiency. Parasite. 27, 43 (2020).

18. Zheng, $X$. et al. Incompatible and sterile insect techniques combined eliminate mosquitoes. Nature. 572 (7767), 56-61 (2019).

19. BEl Resources. Methods in Aedes Research.<https:// www.beiresources.org/Portals/2/VectorResources/
Methods\%20in\%20Aedes\%20Research\%202016.pdf> (2016).

20. Focks, D. A. An improved separator for the developmental stages, sexes, and species of mosquitoes (Diptera: Culicidae). Journal of Medical Entomology. 17 (6), 567-568 (1980).

21. International Atomic Energy Agency. Manual of Dosimetry in Radiotherapy. Technical Reports Series No. 110. IAEA. Vienna (1970).

22. Aldridge, R. L. et al. Gamma-irradiation reduces survivorship, feeding behavior, and oviposition of female Aedes aegypti. Journal of the American Mosquito Control Association. 36 (3), 152-160 (2020).

23. Cianci, D. et al. Estimating mosquito population size from mark-release-recapture data. Journal of Medical Entomology. 50 (3), 533-542 (2013).

24. Knipling, E. F. The basic principles of insect population suppression and management. Washington, DC: United States Department of Agriculture (1979). 